\title{
Comparison of Modified Soret Lenses for Dual Band Integrated Detectors
}

\author{
Alicia E. Torres-García*†, José M. Pérez-Escudero*†, Ramón Gonzalo*†, Iñigo Ederra*† \\ *Depart. Electrical, Electronic and Communication Engineering, Universidad Pública de Navarra, \\ Pamplona, Spain, aliciae.torres@unavarra.es \\ ${ }^{\dagger}$ Institute of Smart Cities, Universidad Pública de Navarra, Pamplona, Spain, inigo.ederra@unavarra.es
}

\begin{abstract}
This paper presents the comparison of different modified Soret lenses suitable for a millimeter and submillimeterwave dualband integrated pixel detectors. The approach is based on the modification of a printed planar Soret lens, designed to operate in the sub-mm range, to obtain an antenna at the millimeter region. Three modifications of a transmissionmode Soret Lens at $850 \mathrm{GHz}$ based on spiral, logarithmic and meander antennas geometries have been analyzed with a combination of Kirchhoffs Diffraction and full-wave simulation methods. The performance of the designs has been experimentally demonstrated in the submillimeterwave band, showing good agreement with simulation results.
\end{abstract}

Index Terms-Soret lens, integrated receivers.

\section{INTRODUCTION}

Multiband antennas can find their aplication in imaging, where they increase the information obtained from the phenomena under inspection [1],[2]. One combination of frequencies that can be of particular interest integrates the millimetre and $\mathrm{THz}$ ranges, since it allows penetration through some materials at millimeter waves while having the higher spatial resolution provided by $\mathrm{THz}$ frequencies. This could be of potential interest for security applications, since the combination of these frequency ranges could enhance detection of concealed threats and, thanks to the possibility of discrimination between different materials, even their identification. This type of application would benefit from compact integrated multiband detectors, since they would allow obtaining in a single measurement information from different frequency ranges.

One solution for this concept was proposed [3], [4]. In them, a Soret lens was created in the low frequency antenna. In this work, we extend the study of this type of detectors to the millimeter wave and $\mathrm{THz}$ bands. The standard Soret lens, working at $800-900 \mathrm{GHz}$, will be approximated by different W-band antenna types. We will compare their performance as lenses at $800-900 \mathrm{GHz}$ range using for the analysis both a fast scalar Kirchhoff's diffraction theory approach and 3D Full-Wave electromagnetic simulations. Finally, the prototypes were fabricated and measured; thus, experimental results are also presented.

\section{DUAL BAND CONFIGURATION}

The proposed dual band detector would be formed by a millimeter wave antenna (in this case working at around
$100 \mathrm{GHz}$ ) which simultaneously acts as a lens for the $\mathrm{THz}$ detectors. This antenna is printed on a dielectric slab, with the $\mathrm{THz}$ detector placed on the opposite face of the slab. Thickness of the slab corresponds to the lens focal length. An schematic is shown in Fig.1).

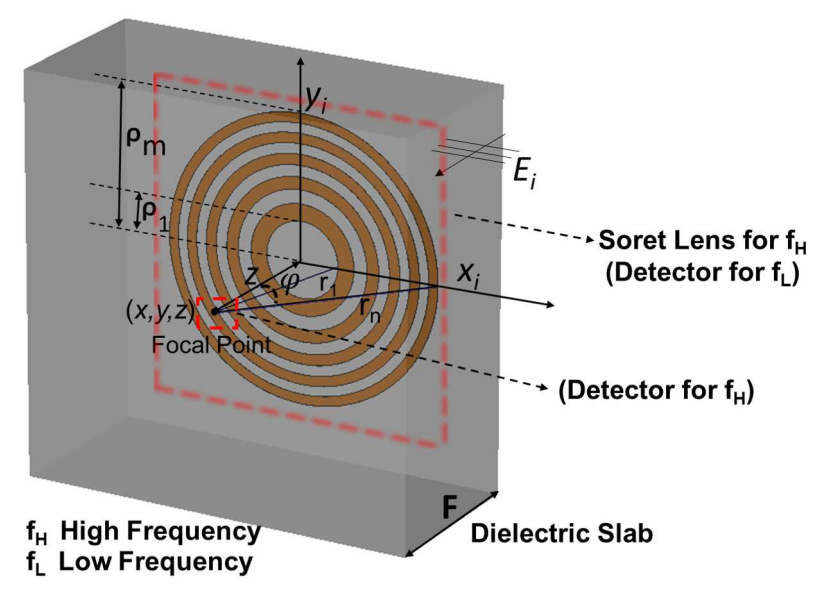

Fig. 1. 3D perspective view of the proposed dual band detector based on a Soret lens.

The millimeter wave antenna is meant to work as a lens, so that it focuses the incoming $\mathrm{THz}$ radiation on the detector. Therefore, it must resemble a planar lens, in this case a Soret lens. This type of lens is formed by alternated opaque and transparent Fresnel zones [5], [6], which are obtained by printed metallic rings, as shown in Fig.1. For a $F$ focal distance, the radius of the $m^{t h}$ ring $\left(\rho_{m}\right)$ is determined by 1 [5]:

$$
\begin{array}{r}
\rho_{m}=\sqrt{m \lambda F+\left(\frac{m \lambda}{2}\right)^{2}} \\
\\
m=1,2, \ldots M_{t}
\end{array}
$$

where $\lambda$ is the operational wavelength (in this case the wavelength in the substrate).

We aim at combining in an integrated configuration two detectors. First, a sub-mmw conventional Soret lens in transmission mode (even numbered zones opaque) is designed to operate at around $850 \mathrm{GHz}$. It will focus the incoming radiation form the air side on the opposite side of the dielectric

(C) 2020 IEEE. Personal use of this material is permitted. Permission from IEEE must be obtained for all other uses, in any current or future media, including reprinting/republishing this material for advertising or promotional purposes, creating new collective works, for resale or redistribution to servers or lists, or reuse of any copyrighted component of this work in other work. 


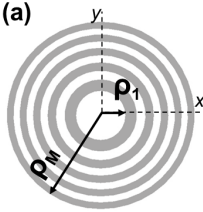

(b)

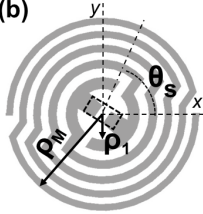

(c)

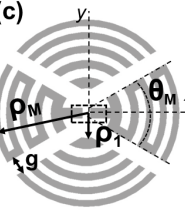

(d)

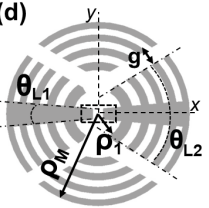

Fig. 2. Schematic of the different approaches to create a modified Soret based on antennas: (a) Conventional 10 zone Soret lens; (b) quasi-spiral antenna; (c) quasi-meander antenna; (d) quasi-log antenna.

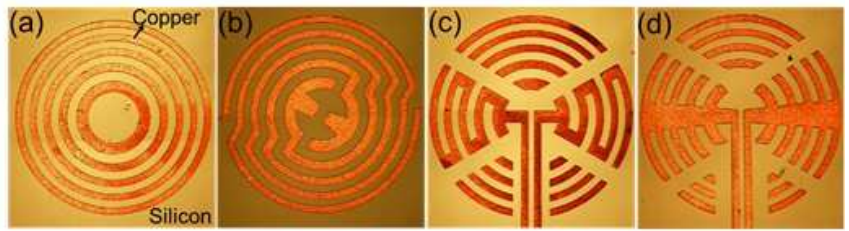

Fig. 3. Fabricated W-Band antennas that can act as Lenses at $850 \mathrm{GHz}$ (a) Conventional 10 zones Soret lens: (b) quasi-spiral antenna, (c) quasi-meander antenna with read-out lines(d) quasi-log antenna with read-out lines.

slab, where the detector would be placed (see Fig.1). In order to accommodate the W-band receiver, this Soret lens is substituted by a mmw antenna which resembles its shape. Three different solutions have been considered:

1) Quasi-spiral antenna

The spiral is formed by connecting consecutive metal rings, see Fig. 2 b).

2) Quasi-meander antenna

In this case half of the lens is left unchanged, whereas the other half creates the meander by connecting consecutive metal rings, as shown in Fig. 2 c).

3) Quasi-logperiodic antenna

This is similar to the meander antenna, but the rings are connected by a central metallic strip, see Fig. 2 d).

The design parameters that can be used in each of these configurations are shown in Fig. 2. In all cases the 10 section Soret lens in Fig. 2 a) was the starting point. In addition, the pads for connection of the detector (e.g. a zero-bias Schottky diode) are also considered. In the case of the meander and logperiodic, the read-out lines have been also considered. These are not drawn in the schematics, but have been included in the manufactured prototypes shown in Fig. 3.

\section{RESULTS}

The performance of these Soret lens antennas was evaluated using Kirchhoff's diffraction theory [7] and full-wave simulations. Good agreement between both results was found.

Fig. 4 shows the Field Gain (defined as the as the squared ratio of the focused field, $E(F)$, and the free-space electric field $E_{0}(F)$, at the focal point F, i.e. $F G[d B]=20 \log _{10}\left(\frac{|E(F)|}{\left|E_{0}(F)\right|}\right)$ and E-field magnitude values at the focal point versus frequency. Two cases have been considered: a semi-infinite silicon substrate and (dashed line) a finite silicon slab with $520 \mu \mathrm{m}$ thickness (solid-line). Both horizontal and vertical polarizations have been considered. The simulations show that (a)

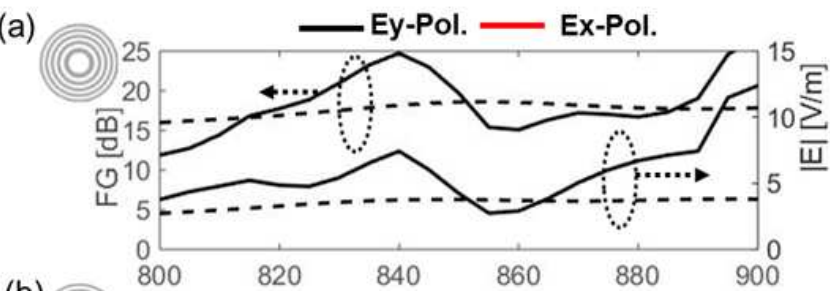

(b)

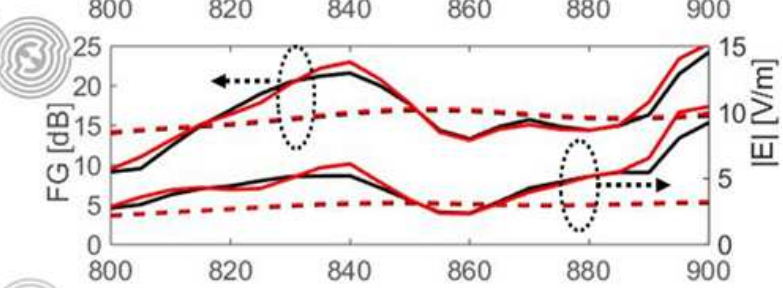

(c)

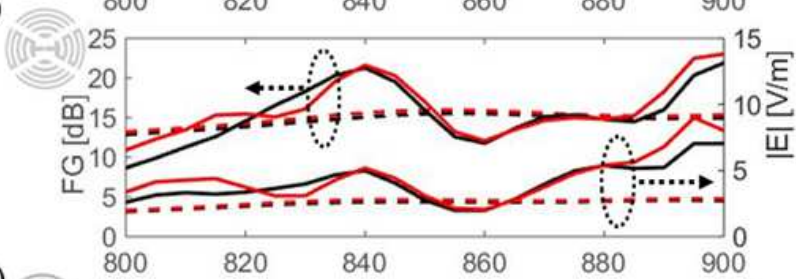

(d)

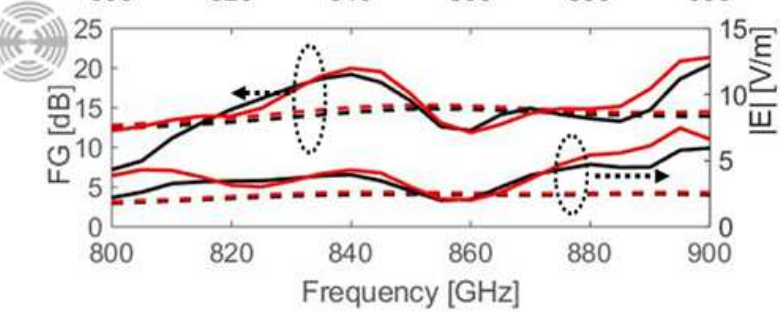

Fig. 4. Focal Gain and E-field magnitude at the focal point ( $x=0, y=$ $0, z=520 \mu \mathrm{m}$ ) vs frequency for infinite silicon substrate (dashed lines) and silicon slab (solid lines) with vertical (black) and horizontal (red) polarized incident wave. (a) Soret Lens, (b) quasi-spiral, (c) quasi-meander and (d) quasi-log models.

the Field Gain decreases with respect the original Soret lens. At $855 \mathrm{GHz}$, the maximum FG for the Soret lens is 18.6 $\mathrm{dB}$, whereas it is $17.1 \mathrm{~dB}$ for the quasi-spiral, $16 \mathrm{~dB}$ for the quasi-meander and $15.3 \mathrm{~dB}$ in the quasi-log design for infinite substrate. The quasi-spiral design is the one that bears largest resemblance to the performance of the conventional lens whilst the quasi-meander and quasi-log approaches reach comparable performance. The finite substrate creates a standing-wave pattern, which allows having larger FG than in the semi-infinite case. These maxima are nearly $25 \mathrm{~dB}$ for the Soret lens and $22,20.5$ and $20 \mathrm{~dB}$ for the three other cases. No significant differences between polarizations can be observed.

To validate the performance of these solutions the prototypes shown in Fig. 3 were manufactured at the Public University of Navarra's facilities. They were made by e-beam evaporation of $1 \mu \mathrm{m}$ of $\mathrm{Cu}$ on a $400 \mu \mathrm{m}$ thick high resistivity silicon waffer.

The experimental characterization of the lenses was performed with an $\mathrm{AB}$ Millimetre quasi-optical vector network analyzer with the setup shown in Fig. 5. The prototypes were illuminated by a $25 \mathrm{dBi}$ gain WR 1.2-band diagonal horn antenna placed at distance $\mathrm{L}=70 \mathrm{~mm}$. At this distance, for a 
(a)

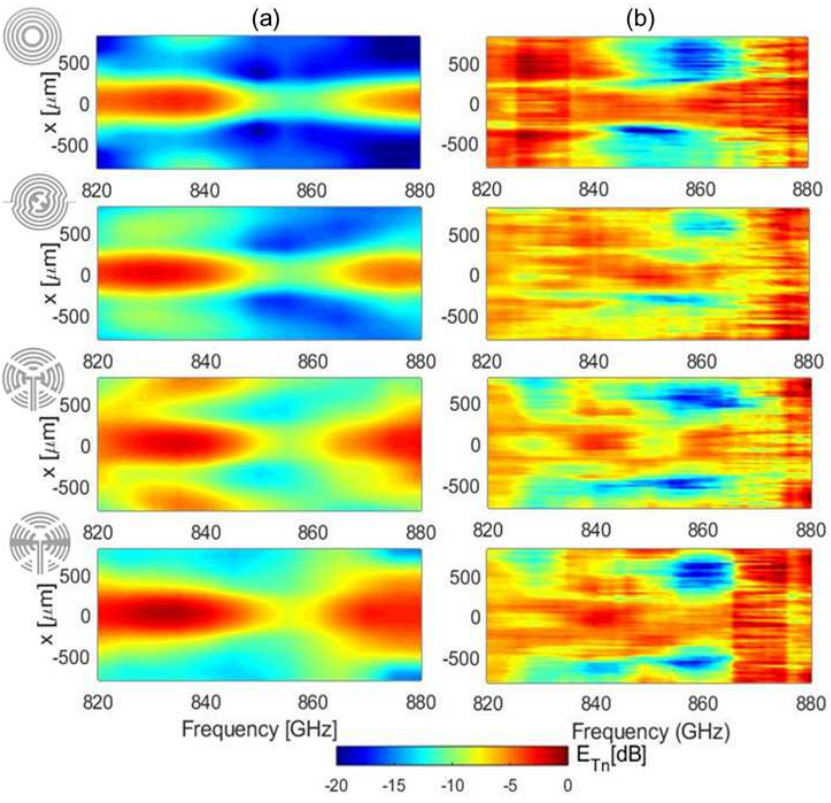

Fig. 6. Normalized power distribution along $\mathrm{x}-$ axis at $z=540 \mu \mathrm{m}$ for the frequency range $820880 \mathrm{GHz}$ : (a) Simulation including the effect of the detection probe and (b) experimental results.

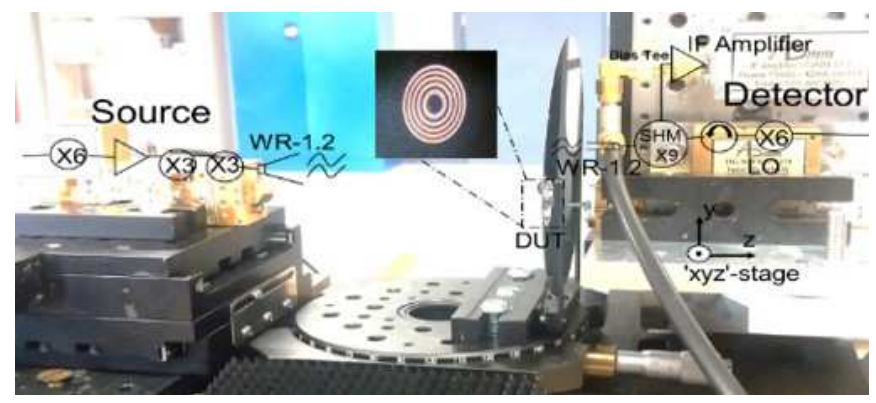

Fig. 5. Photograph of the experimental setup for the characterization of the lenses performance.

central operational frequency of $850 \mathrm{GHz}\left(\lambda_{0} \sim 350 \mu \mathrm{m}\right)$, uniform illumination of the lens is ensured. To scan the focal plane field distribution, a rectangular WR-1.2 probe was moved from -800 to $800 \mu \mathrm{m}$ along the $\mathrm{x}$ and $\mathrm{y}$-axis in $20 \mu \mathrm{m}$ steps. Note that the focal distance of the lens corresponds to the dielectric substrate thickness. Therefore, the probe detector was placed as close as possible to the dielectric-air interface. However, a minimum separation between must be kept to allow the movement of the probe. Thus, the simulated field distributions correspond to a plane separated $20 \mu \mathrm{m}$ of the dielectric.

The simulations corresponding to this configuration are shown in Figure 6. The clear focus observed in the nominal
Soret lens is slightly distorted for the antenna approximations. In this simulations the effect of the waveguide probe used in the experimental characterization has been included. The experimental results, shown in the left column are normalized with the response of a silicon wafer. Similar trends than in the simulations can be observed.

Despite the minor shift in frequency, all models exhibit similar bandwidth performance in the designed frequency range. The experimental results display the presence of the focus for all prototypes with a better definition in the Soret lens case. The very small wavelength, and the small distance between the probe and the silicon wafer, due to the focal point localization exactly at the dielectric-air interface, make this measurement especially prone to present ripples in the experimental data caused by the excitation of standing waves between the lens and the probe. For the same reason, the measurement is also very sensitive to any lens inclination.

\section{CONCLUSION}

In this paper, different antenna configurations able to function as Soret lens have been analyzed. In particular, spiral, logarithmic and meander antennas geometries have been analyzed with a combination of Kirchhoffs Diffraction and full-wave simulation methods. The performance of the designs has been experimentally demonstrated in the submillimeterwave band, showing good agreement with simulation results.

\section{ACKNOWLEDGMENT}

The authors would like to thank financial support by the Spanish Ministry of Science and Innovation, Project No. TEC2013-47753-C3-1-R, TEC2014-61817-EXP and TEC2016-76997-C3-1-R.

\section{REFERENCES}

[1] M. Kowalski, M. Kastek, H. Polakowski, N. Palka, M. Piszczek, and M. Szustakowski, Multispectral concealed weapon detection in visible, infrared, and terahertz, Proceedings of the SPIE, 91020T (2014), vol. 8, no. 10, p. 91020T, 2014.

[2] R. Appleby and H. B. Wallace, Standoff detection of weapons and contraband in the $100 \mathrm{GHz}$ to $1 \mathrm{THz}$ region, IEEE Trans. Antennas Propag., vol. 55, no. 11 I, pp. 29442956, 2007.

[3] F. J. Gonzalez, B. Ilic, J. Alda and G. D. Boreman, "Antenna-coupled infrared detectors for imaging applications," IEEE Journal of Selected Topics in Quantum Electronics, vol. 11, no. 1, pp. 117-120, Jan.-Feb. 2005.

[4] D. Etayo, I. Ederra, R. Gonzalo, "Analysis of square Fresnel Zone Plate Lens for dual band detectors", Journal of Infrared Millimeter and Terahertz Waves, Vol. 35, pp. 525- 535, 2014.

[5] H. D. Hristov, Fresnel Zones in Wireless Links, Zone Plate Lenses and Antennas, vol. 1. 2000.

[6] I. V. Minin and O. V. Minin, Reference Phase in Diffractive Lens Antennas: A Review, J. Infrared, Millimeter, Terahertz Waves, vol. 32, no. 6, pp. 801822, 2011.

[7] A. E. Torres-Garca, B. Orazbayev, I. Ederra, and R. Gonzalo, IR-Fresnel zone plate lens acting as $\mathrm{THz}$ antenna, 10th Glob. Symp. MillimeterWaves, GSMM 2017, no. 1, pp. 129131, 2017. 